\title{
Impact of scribes on patient interaction, productivity, and revenue in a cardiology clinic: a prospective study
}

This article was published in the following Dove Press journal:

ClinicoEconomics and Outcomes Research

8 August 2013

Number of times this article has been viewed

\author{
Alan J Bank' \\ Christopher Obetz ${ }^{2}$ \\ Ann Konrardy ${ }^{2}$ \\ Akbar Khan' \\ Kamalesh M Pillai' \\ Benjamin J McKinley' \\ Ryan M Gage' \\ Mark A Turnbull' \\ William O Kenney' \\ 'United Heart and Vascular Clinic, \\ St Paul, MN, USA; ${ }^{2}$ Abbott \\ Northwestern Hospital, \\ Minneapolis, MN, USA
}

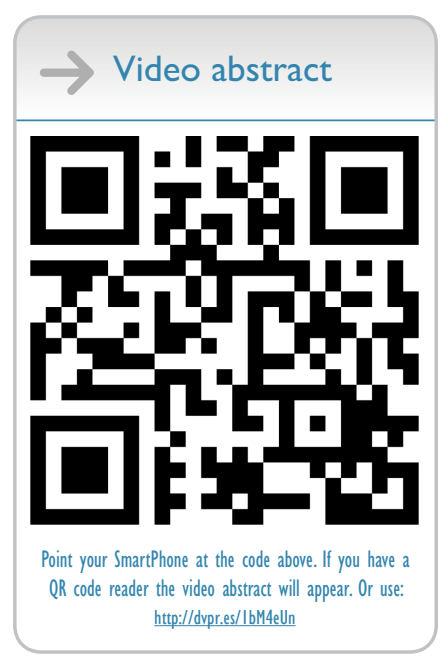

Correspondence: Alan J Bank United Heart \& Vascular Clinic, 225 N Smith Ave, Suite 400 St Paul, MN 55102, USA

Tel + I 65I 24I 2819

Fax + I 65I 24I 2818

Email alan.bank@allina.com
Objective: Scribes have been used in the emergency department to improve physician productivity and patient interaction. There are no controlled, prospective studies of scribe use in the clinic setting.

Methods: A prospective controlled study compared standard visits (20 minute follow-up and 40 minute new patient) to a scribe system (15 minute follow-up and 30 minute new patient) in a cardiology clinic. Physician productivity, patient satisfaction, physician-patient interaction, and revenue were measured.

Results: Four physicians saw 129 patients using standard care and 210 patients with scribes during 65 clinic hours each. Patients seen per hour increased $(P<0.001)$ from $2.2 \pm 0.3$ to $3.5 \pm 0.4$ ( $59 \%$ increase) and work relative value units (wRVU) per hour increased $(P<0.001)$ from $3.5 \pm 1.3$ to $5.5 \pm 1.3(57 \%$ increase $)$. Patient satisfaction was high at baseline and unchanged with scribes. In a substudy, direct patient contact time was lower $(9.1 \pm 2.0$ versus $12.9 \pm 3.4$ minutes; $P<0.01$ ) for scribe visits, but time of patient interaction (without computer) was greater $(6.7 \pm 2.1$ versus $1.5 \pm 1.9$ minutes; $P<0.01)$. Subjective assessment of physician-patient interaction $(1-10)$ was higher $(P<0.01)$ on scribe visits $(9.1 \pm 0.9$ versus $7.9 \pm 1.1$ ). Direct and indirect (downstream) revenue per patient seen was $\$ 142$ and $\$ 2,398$, with $\$ 205,740$ additional revenue generated from the 81 additional patients seen with scribes.

Conclusion: Using scribes in a cardiology clinic is feasible, produces improvements in physician-patient interaction, and results in large increases in physician productivity and system cardiovascular revenue.

Keywords: physician productivity, medical economics, patient satisfaction, physician-patient interaction, scribe

\section{Introduction}

The transition to electronic medical records (EMR) has created increased demands on physician time. It also, however, offers opportunities to improve the efficiency of care and the physician-patient interaction. An approach used to improve efficiency and quality of care in some emergency departments (ED) has been the use of scribes. ${ }^{1-3}$ In one study, the use of scribes increased ED patients seen per hour by 0.8 , and work relative value units (wRVUs) generated per hour by $2.4 .{ }^{3}$ Scribes are typically students planning to work in the medical field who assist physicians with the clerical aspects of patient care. Although some physicians have started using scribes in the outpatient setting, there is little data on the impact of scribes on physician-patient interaction, physician productivity, or financial ramifications. Therefore, we performed a 
prospective controlled trial comparing standard care (without scribes) to patient care using a scribe in a large outpatient cardiology clinic.

\section{Methods}

\section{Clinic and electronic medical record}

This study was performed at United Heart and Vascular Clinic (UHVC), a single outpatient clinical setting. UHVC is a cardiology group in St Paul, MN, USA owned by a large health care organization, Allina Health. There are 25 cardiologists and four nurse practitioners who provide care in all main areas of cardiology except heart transplantation. UHVC physicians started using an EMR (Epic Systems Corporation, Verona, WI, USA) approximately 4 years ago in the inpatient setting. The outpatient version of this EMR was fully integrated and used in the clinic setting 4 months prior to this study.

\section{General study design}

Four experienced cardiologists volunteered to participate in the study and were assigned approximately equal numbers of clinic hours on control and scribe days. On standard clinic days, patients were scheduled at 20 minutes for follow-up and 40 minutes for new patient visits. Every 4 hours, one follow-up slot was left unscheduled for physicians to catch up with dictation/documentation (standard clinic practice). The open 20-minute slot is part of standard clinic practice because when our clinic began using an EMR, physicians were unable to see patients at a pace of one every 20 minutes without falling far behind in their visits. On scribe days, patients were scheduled at 15 minutes for follow-up and 30 minutes for new patients. No slots were intentionally left unscheduled. This study was performed in accordance with national regulations and was deemed exempt from institutional review board approval for human subjects.

\section{Scribes}

This study was performed in collaboration with Emergency Care Consultants, a professional corporation providing medical scribe services. A scribe with 6 years' experience as an ED scribe, but no experience in cardiology or in clinic, participated. The scribe received training for 3 hours on clinic procedures and cardiology-specific issues. The scribe arrived 1 hour prior to a 4-hour clinic session to review records and generate preliminary notes. A template created by each physician was used to populate from the EMR the problem list, past medical and surgical history, medications, allergies, social and family history, and relevant laboratory and test data. The scribe summarized pertinent clinic visits, hospitalizations, and relevant past medical history. During the patient visit, the scribe modified the progress note and searched for additional medical information at the physician's request. Other tasks performed with direction from the cardiologist included: entering diagnoses, revising the problem list, completing the follow-up request form, typing patient instructions, documenting level of service, and completing the after-visit summary. Following the visit, the physician reviewed the notes, made modifications, and entered any new orders.

\section{Patient satisfaction surveys}

Patients completed a standard questionnaire. They rated their visit with the doctor as follows: 1) willingness to listen carefully to you, 2) taking time to answer your questions, 3 ) explaining things in a way you can understand, 4) thoroughness of the examination, 5) instructions regarding follow-up care, and 6) courtesy of the doctor. They rated overall satisfaction with: 1) the practice, 2) the quality of their medical care, and 3) overall rating of care from their doctor that day. All responses were graded by using a five-point Likert-type scale with the following categories: excellent (5), very good (4), good (3), fair (2) and poor (1).

\section{Time-motion analysis}

A performance improvement manager with extensive experience consulting on physician-patient interaction observed one physician (AJB) during 4 hours of designated scribe visits and 4 hours of control clinic visits. The physician who participated in this substudy had been using the EMR for $\sim 4$ years in the inpatient setting and 4 months in the outpatient setting, and was experienced and facile with its use. The performance improvement manager recorded time performing the following tasks: 1) prior to the visit: preparation of the clinic note, review of the patient record; 2) during the visit: interaction with the patient with and without the computer, physical examination; and 3) after the visit: documentation of the visit (typing or dictating). Clinic visits were graded by the performance improvement manager on physician-patient interaction quality using a scale of 1 (low) to 10 (high). A single subjective grade was used and based on the observer's overall impression of the physician's attentiveness, knowledge of patient's history, use of empathy and open-ended questions, courtesy and respect, repetition of key points to the patient, and clarity of the treatment plan explanation.

\section{Physician productivity}

Physician productivity was measured by patients seen and wRVUs per hour. The wRVUs were based on the level of 
service for each patient visit and reviewed by coders who ensured that the documentation justified the level of service. Since new patients were allotted (and typically require) twice as much time as follow-up patients, each new patient visit was counted as two visits.

\section{Financial measurements}

Direct clinic revenue was measured for control and scribe visits. Total downstream revenue was defined as all cardiovascular (CV) revenue generated in the 2 months following each clinic visit. Downstream revenue was separated into three categories: professional services (physician billing for inpatient visits, tests, and procedures), outpatient (technical revenue for outpatient tests and procedures) and hospital inpatient revenue. Total revenue in each category was divided by the number of patients to calculate the average revenue generated per patient. Additional revenue generated through the increased number of patients seen using scribes was calculated as the average revenue per patient multiplied by the number of additional patients. The cost of using scribes was $\$ 25$ per hour, which included time preparing the charts prior to patient visits, participating in the clinic visits, and completing documentation after visits.

\section{Statistics}

Productivity measures, physician-patient interaction scores, patient satisfaction scores, and time spent performing different activities for control and scribe days were calculated and reported as mean \pm standard deviation. $P$-values were calculated using unpaired $t$-tests. $P$-values $<0.05$ were considered significant.

\section{Results}

Sixty-five hours of clinic care were provided on control and scribe days each. Productivity measures are shown in Figure 1. On control days, 114 follow-up and 15 new patients were seen. On scribe days, 191 follow-up and 19 new patients were seen. The percentage of patients that were new as opposed to follow-up was similar on control $(11.6 \%)$ and scribe $(9.0 \%)$ days. Average direct wRVUs generated per new patient on control $(2.67 \pm 0.74)$ and scribe $(2.71 \pm 0.57)$ visits were similar $(P=0.89)$. Average direct wRVUs generated per follow-up patient on control $(1.64 \pm 0.57)$ and scribe $(1.60 \pm 0.47)$ visits $(P=0.49)$ were also similar. Counting new patients as two, patients per hour increased $59 \%$ from $2.2 \pm 0.4$ on control days to $3.5 \pm 0.4$ on scribe days $(P<0.001)$. Total wRVUs per hour increased $57 \%$ from 227 (3.5 \pm 1.3 per hour) on control days to 356 $(5.5 \pm 1.3$ per hour) on scribe days $(P<0.001)$.

Direct clinic revenue for control and scribe visits was $\$ 48,255$. Total indirect (downstream) physician services revenue was $\$ 185,569$. Total indirect outpatient revenue was \$269,218 from 116 different encounters. Total indirect hospital revenue was $\$ 358,393$ from 16 hospital admissions Average revenue generated per patient was: $\$ 142$ (direct), $\$ 547$ (indirect physician services), \$794 (indirect outpatient), and $\$ 1,057$ (indirect hospital inpatient). Additional revenue generated by the additional 81 patients seen using scribes

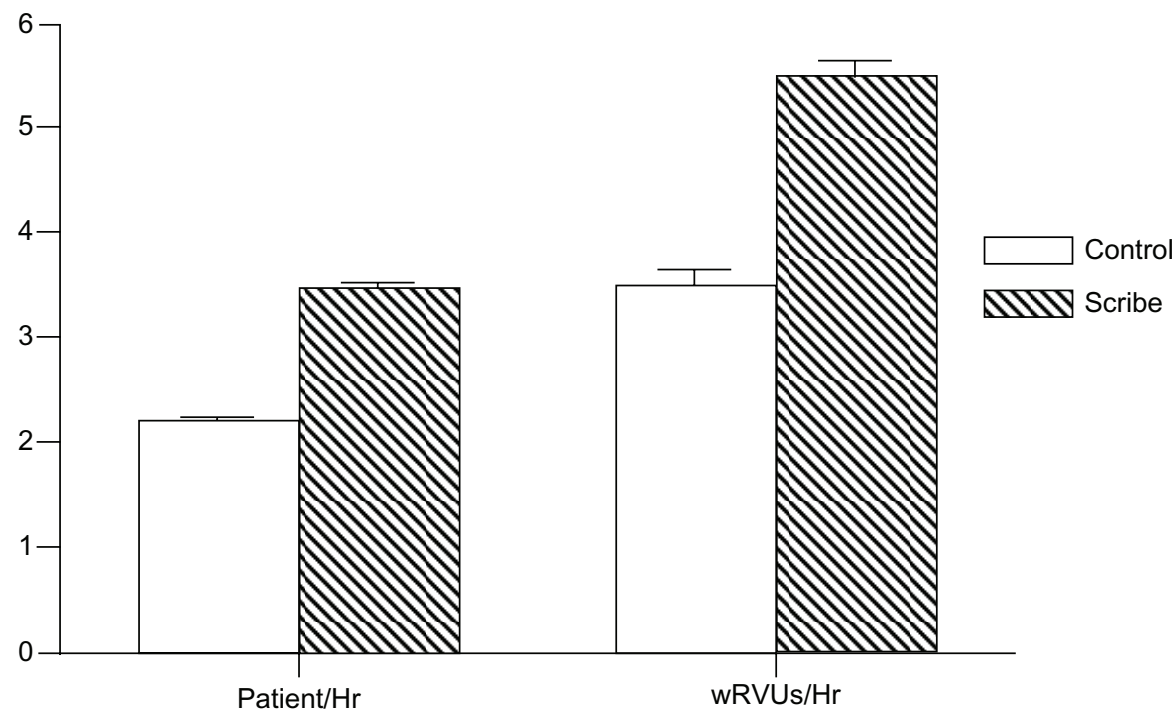

Figure I Physician productivity.

Note: Productivity measured as number of patients seen per hour and number of wRVUs generated per hour on control and scribe clinic visits. Abbreviations: $\mathrm{Hr}$, hour; wRVUs, work relative value units. 
was $\$ 11,502$ (direct), $\$ 44,307$ (indirect physician services), $\$ 64,314$ (indirect outpatient), and $\$ 85,617$ (indirect hospital inpatient). Additional revenue was $\$ 2,540$ per patient and $\$ 205,740$ total. The only additional direct expense was for leasing the scribe at $\$ 25$ per hour for 82 hours $(\$ 2,050)$. The average cost per visit of using scribes was $\$ 8.95$ for follow-up visits and $\$ 19.90$ for new patient visits.

Figure 2 shows patient satisfaction data for the nine questions. Patient satisfaction was very high on control days, and remained high and unchanged on scribe days. The four cardiologists who participated in the study were interviewed and all felt that scribe use resulted in reduced tedious paperwork, improved efficiency, and more time in direct interaction with the patient. We performed a timemotion analysis on nine control patient visits and 14 scribe visits. Table 1 shows the mean time spent performing patient visit tasks. Figure 3 shows this data in a visual timeline. Control visits averaged 24.7 minutes and scribe visits averaged 15.5 minutes. The average time required to complete a scribe visit (including documentation) was 37\% shorter than that needed for a control visit. Previsit, visit, and postvisit times were all significantly lower when using scribes. Despite reduced physician time in the patient room, time spent in direct patient interaction (without using the computer) was over fourfold greater on scribe visits. Overall quality of the physician-patient interaction was also significantly $(P<0.01)$ better on scribe $(9.1 \pm 0.9)$ versus control visits $(7.9 \pm 1.1)$.

\section{Discussion}

To our knowledge, this is the first prospective controlled study assessing the clinical and financial aspects of using scribes in an outpatient clinic. We demonstrate that the use of a medical scribe in a large cardiology outpatient clinic resulted in a marked improvement in physician productivity as well as large increases in direct and downstream revenue. This was achieved at low cost, without major disruption in patient flow, with unchanged high patient satisfaction, increased direct physician-patient interaction time, and improved quality of physician-patient interaction.

\section{Physician productivity}

EMRs are increasingly being implemented throughout the US healthcare system and can have significant beneficial effects on patient safety, health care quality, and health care delivery costs. However, the impact of EMRs on physician productivity is of great concern. In addition, the increased paperwork and time required to learn and use EMRs may contribute to the problem of physician burnout. ${ }^{4}$ In the Medical Economics Electronic Health Record Survey, 100\% of the physicians questioned listed "excessive use of their time and their staff's time" as their greatest concern while they prepared to implement EMRs. ${ }^{5}$ In a survey of 1,000 physicians, 54\% agreed or strongly agreed that EMRs slow down the doctor during patient exams. ${ }^{6}$ A study of 87 internal medicine, family practice, and pediatric physicians showed that physician productivity dropped immediately after implementation of an EMR but began to recover in a few months, with average productivity 6 months later at, or slightly below, baseline. ${ }^{7}$

The impact of scribes on physician productivity has been studied in the ED. ${ }^{3}$ Patients seen per hour increased by 0.8 , and wRVUs per hour increased by 2.4 , with no significant change in time to discharge. Our study assessed the impact of scribes on physician productivity in a medical subspecialty

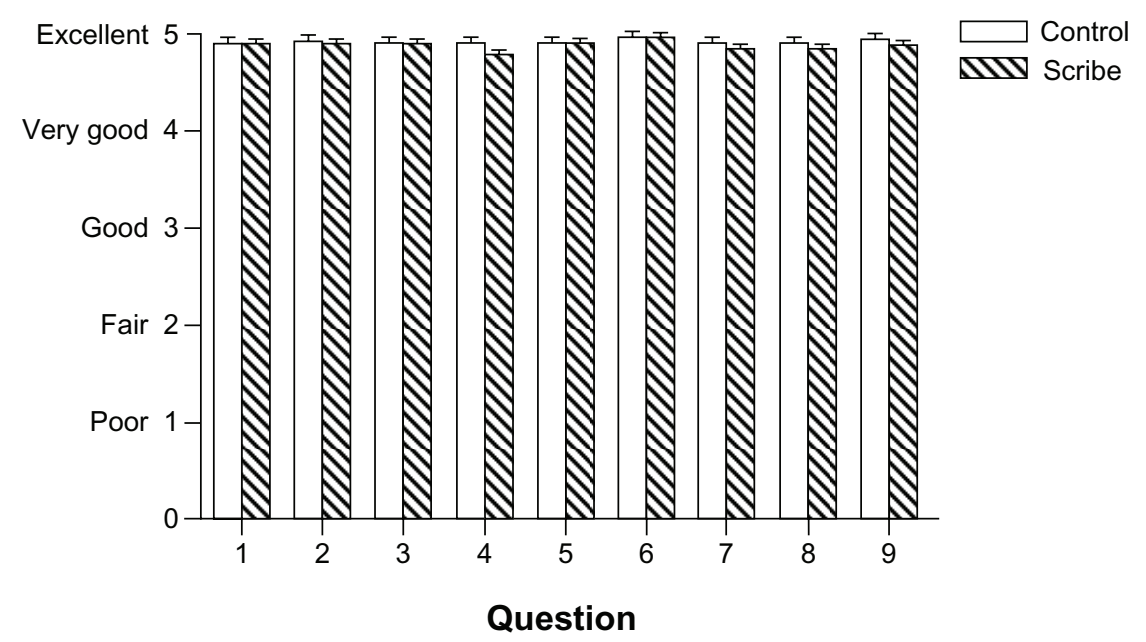

Figure 2 Patient satisfaction.

Notes: Patient satisfaction as measured by questionnaire after control and scribe clinic visits. They rated their visit with the doctor as follows: I) willingness to listen carefully to you, 2) taking time to answer your questions, 3) explaining things in a way you can understand, 4) thoroughness of the examination, 5) instructions regarding follow-up care, 6) courtesy of the doctor. They rated overall satisfaction with: 7) the practice, 8) the quality of their medical care, and 9) overall rating of care from their doctor. 
Table I Time-motion analysis of physician clinic activities

\begin{tabular}{lrcl}
\hline & Control & Scribe & P-value \\
\hline Chart preparation (I) & $3.7 \pm 1.4$ & $0 \pm 0$ & $<0.0 \mathrm{I}$ \\
Chart review (2) & $1.2 \pm 0.5$ & $2.8 \pm 1.9$ & 0.02 \\
MD/patient interaction - no & $1.5 \pm 1.9$ & $6.7 \pm 2.1$ & $<0.0 \mathrm{I}$ \\
computer (3) & & & \\
MD/patient interaction - with & $9.3 \pm 2.6$ & $0.5 \pm 0.3$ & $<0.0 \mathrm{I}$ \\
computer (4) & & & \\
Physical exam (5) & $2.1 \pm 1.0$ & $2.0 \pm 0.7$ & $0.6 \mathrm{I}$ \\
Dictation and postvisit note (6) & $7.0 \pm 3.8$ & $4.2 \pm 2.1$ & 0.04 \\
Previsit (I + 2) & $4.9 \pm 1.9$ & $2.8 \pm 1.9$ & 0.02 \\
Visit (3-5) & $12.9 \pm 3.4$ & $9.1 \pm 2.0$ & $<0.0 \mathrm{I}$ \\
Postvisit (6) & $7.0 \pm 3.8$ & $4.2 \pm 2.1$ & 0.04 \\
\hline
\end{tabular}

Note: Data presented as minutes $\pm S D$.

Abbreviations: MD, Doctor of Medicine; SD, standard deviation.

outpatient clinic, where the issues and impact of using scribes may differ. In particular, patients in an ED are not scheduled and are seen at the physician's discretion based on severity of illness - a very different patient flow than in a clinic. We demonstrate very large increases in physician productivity. Based on our scheduling design, we planned to increase physician productivity by $45 \%$. In fact, we ended up with a $57 \%-59 \%$ increase. We did not see the number of patients projected due to some patient slots not filled, or patients missing or rescheduling their visits. In our standard clinical practice with 20-minute follow-up visit slots, we were at maximum productivity, as evidenced by the fact that we had to leave an open 20-minute slot every 4 hours or else physicians could not see patients and complete their visit within the allotted time frame. It is important to note that the use of scribes produced a true increase in productivity: patient visits were on time as scheduled, documentation was mostly or completely finished within the clinic time frame, and physicians were not working after clinic to complete documentation. This improved productivity was confirmed by the time-motion substudy, which demonstrated a $37 \%$ reduction in patient visit time with a scribe. The time-motion study not only was entirely consistent with the overall study findings on productivity, but also demonstrated why scribe visits were more efficient. Physicians spent less time gathering, collating, and documenting data, which reduced time spent performing these tasks prior to, during, and after each visit. This enabled the physician to spend more time in direct face-to-face interaction with the patient despite the shorter time scheduled and needed for each visit. A byproduct of increased physician productivity is improved patient access to care. Implementation of models such as the one described here on a larger scale can potentially significantly improve patient access in the face of a projected shortage of physicians. ${ }^{4}$

\section{Patient satisfaction and physician- patient interaction}

Communication between the doctor and the patient has a large impact on patient satisfaction, medication adherence, conflict resolution, and clinical outcomes. Many physicians have expressed concern about the use of an EMR on the

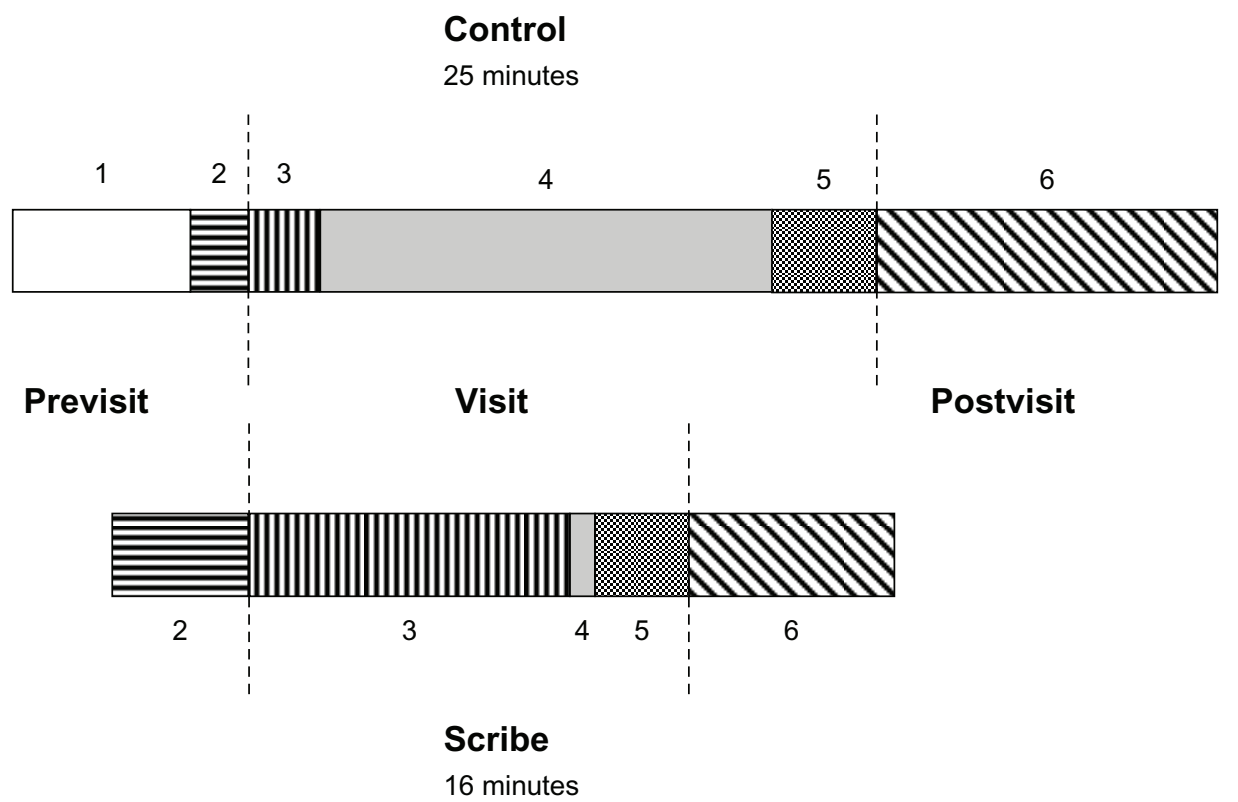

Figure 3 Timeline of physician work activities on control and scribe days.

Notes: Average physician time spent performing the following components of control and scribe patient visits: I = chart preparation, $2=$ chart review, $3=$ physician-patient interaction without using the computer, $4=$ physician-patient interaction with the computer, $5=$ physical examination, $6=$ postvisit documentation and orders. 
provider-patient relationship in the exam room. Use of an EMR results in less psychosocial discussion, less attention to the patient's agenda, ${ }^{8,9}$ and a shift from a conversational to a blocked style of communication. ${ }^{10}$ One approach to these problems is the use of a scribe, which allows the physician to focus on the patient rather than the computer. There is little data on the effects of scribes on patient-doctor interaction. Koshy et al evaluated whether the use of scribes would alter physician and/or patient satisfaction in an ambulatory academic urology setting. ${ }^{11}$ They demonstrated high patient satisfaction (93\%), similar to that found without a scribe (87\%), in a clinic setting where many sensitive issues were discussed.

Patient satisfaction in our study was very high on control and scribe days. Based on written and verbal comments, the vast majority of patients were either neutral toward or liked the scribe system. A number of patients commented to clinic staff about the benefit of having the physician's full attention without distraction from the computer. Clinic staff noted that patient flow improved when using scribes (despite increased numbers of patients being seen), and a number of patients commented that they were being seen in a more timely fashion. Additionally, the quality of the physicianpatient interaction, assessed by an experienced performance improvement manager, was significantly improved on scribe visits, and over 5 minutes of extra time in face-to-face interaction with the patient was noted.

\section{Physician satisfaction}

The addition of a scribe in the exam room, and the associated changes in patient flow, clinic processes, and physicianpatient interaction, is a major change in the traditional clinic visit. Based on patient questionnaires, discussions with ED physicians who have used scribes, and interviews with the physicians in this study, the above changes were beneficial. The main concern of several physicians in the pilot study was that they felt rushed during the 15-minute patient visits (not dissimilar to complaints during standard care with an ERM). This concern about time stress is not surprising, since their productivity was about $58 \%$ higher. This was likely too large a change in too short a time frame. We anticipate adjusting schedules to increase productivity by $10 \%-30 \%$ once the clinic and physicians adapt to the scribe system. We hypothesize that many physicians will have a marked increase in job satisfaction for a number of reasons, including less paperwork, improved patient access to their care, improved physician-patient interaction during clinic visits, reduced time spent before and after clinic, and improved productivity-based pay.

\section{Revenue and cost}

The average clinic visit (90\% follow-up and 10\% new patient) generated $\$ 142$ in direct revenue to the system and \$2,398 in indirect or downstream CV system revenue. There was a wide variation in the downstream revenue generated per patient. For our calculations, we used all $\mathrm{CV}$ revenue generated during the 2 months after each patient visit, assuming this revenue was generated due to tests and procedures ordered at the visit. It is possible that some of this revenue would have been generated even if the patient was not seen in clinic (eg, a patient hospitalized with a new myocardial infarction within 2 months of a clinic visit). The increased revenue generated was a direct result of improved productivity (more patients seen per hour) and not a result of any increase in coding or billing level when using scribes, as shown by the similar wRVUs generated for control and scribe follow-up visits and control and scribe new patient visits.

The cost of implementing a scribe system in this study was quite low. Direct costs for the entire study, including scribe salary/benefits and payments to the company that leased the scribes to us (for training, scheduling, credentialing, etc), were only $\$ 2,050$. The cost of $\$ 25$ per hour is comparable to the cost of transcription. Although we did not need additional computers or personnel to handle logistic issues (scheduling, checking in, and rooming the increased number of patients) in this small pilot study, implementation of this change clinic-wide would likely entail changes in infrastructure, clinic processes, and personnel, with associated costs.

\section{Financial implications}

The financial implications of instituting this change in outpatient care delivery are substantial. Physician services make up $21 \%$ of health expenditures in the United States. ${ }^{12}$ In addition, physicians are highly compensated individuals who are being asked to do more with declining reimbursement. Any change that improves physician productivity and efficiency (without impairing quality or physician or patient satisfaction) should have significant financial benefits for both physicians and for the health care system as a whole. We performed an estimate of the financial impact of utilizing a scribe system for all physicians at our clinic. In the current fee-for-service environment, if each physician in our clinic saw two additional follow-up patients daily ( $9 \%$ increase in productivity), the direct and indirect cardiovascular revenue generation would be approximately $\$ 5.4$ million. Since we have a significant backlog of patients waiting to be seen in our clinic, this revenue would accrue because patients could 
be seen in a more timely fashion and receive appropriate care, not as a result of seeing patients more frequently or performing excessive testing. In an accountable care organization system, the economic benefit would still be present and likely substantial. Improved productivity would allow the same number of physicians to manage a larger patient pool (hence more dollars in the pool) or a reduced number of physicians to manage the same patient pool. In either event, the cost of care per patient is reduced (as long as physicians practice the same way with or without scribes).

\section{Limitations}

This study was performed utilizing a single experienced scribe and four physicians. This allowed for consistency across physicians and minimized the cost of training multiple scribes. We have subsequently trained and utilized six scribes and are increasing the number of physicians using scribes, with good results. The direct revenue measurements were accurate. The indirect revenue was an estimate, and certainly could be higher or lower than calculated. The indirect revenue generated from increased productivity can vary significantly depending upon a number of factors, including physician practice style, patient mix, insurance mix, and medical or surgical specialty. Our substudy was small and involved one physician seeing a total of 23 patients. However, the findings were entirely consistent with our overall study, and also internally consistent across visits such that we only needed to study a relatively small number of patient visits to demonstrate statistically significant benefits with respect to time spent overall and in various components of the visit using scribes. Our study was limited in size and scope, and the effects of this outpatient care delivery model on physicians, patients, and health care systems require further investigation.

\section{Conclusion}

We describe the first controlled prospective trial of scribe use in an outpatient clinical setting. We demonstrate that scribe use in our cardiology clinic is feasible and results in no change in baseline high patient satisfaction, but an improved physician-patient interaction. Physician productivity is markedly increased, as is direct and estimated indirect CV revenue. Approaches such as this that improve physician productivity may have large beneficial impacts on physician job satisfaction, EMR adoption rate, patient access to care, and the cost of delivering care in an increasingly challenging financial healthcare environment.

\section{Acknowledgments}

The authors thank Susan Antol, Jennifer Wolf, Jay Scott, Terry Grilz, Terri Proulx, and Trisha Bonn for assistance with this study.

\section{Disclosure}

No funding was received for this study. Dr Obetz manages the scribe service for Emergency Care Consultants, the company that trained and leased the scribes. The authors report no further conflicts of interest.

\section{References}

1. Allred RJ, Ewer S. Improved emergency department patient flow: five years of experience with a scribe system. Ann Emerg Med. 1983;12(3): 162-163.

2. Scheck A. The next big thing: medical scribes : Scribes push emergency medicine closer to adoption of electronic medical records. Emerg Med News. 2009;31(2):13-16.

3. Arya R, Salovich DM, Ohman-Strickland P, Merlin MA. Impact of scribes on performance indicators in the emergency department. Acad Emerg Med. 2010;17(5):490-494.

4. Shanafelt TD, Boone S, Tan L, et al. Burnout and satisfaction with work-life balance among US physicians relative to the general US population. Arch Intern Med. 2012;172(18):1377-1385.

5. Medical Economics EHR Study Update. Med Econ. April 10, 2012:10.

6. Sermo. $59 \%$ of physicians think the quality of medicine in America will decline in the next five years according to athenahealth and Sermo "Physician Sentiment Index" [press release]. Watertown, MA and Cambridge, MA: Sermo; March 25, 2010. Available from: http://www. sermo.com/news/press-releases/16. Accessed November 1, 2011.

7. Bhargava HK, Mishra AN. Electronic medical records and physician productivity: evidence from panel data analysis [webpage on the Internet]. Available from: http://dx.doi.org/10.2139/ssrn.1952287. Accessed November 1, 2011.

8. Makoul G, Curry RH, Tang PC. The use of electronic medical records: communication patterns in outpatient encounters. J Amer Med Inform Assoc. 2001;8(6):610-615.

9. Margalit RS, Roter D, Dunevant MA, Larson S, Reis S. Electronic medical record use and physician-patient communication: an observational study of Israeli primary care encounters. Patient Educ Couns. 2006;61(1):134-141.

10. Warshawsky SS, Pliskin JS, Urkin J, et al. Physician use of a computerized medical record system during the patient encounter: a descriptive study. Comput Methods Programs Biomed. 1994;43(3-4):269-273.

11. Koshy S, Feustel PJ, Hong M, Kogan BA. Scribes in an ambulatory urology practice: patient and physician satisfaction. J Urol. 2010;184(1): 258-262.

12. Hartman M, Martin A, McDonnell P, Catlin A, National Health Expenditure Accounts Team. National health spending in 2007: slower drug spending contributes to lowest rate of overall growth since 1998. Health Aff (Millwood). 2009;28(1):246-261. 


\section{Publish your work in this journal}

ClinicoEconomics \& Outcomes Research is an international, peerreviewed open-access journal focusing on Health Technology Assessment, Pharmacoeconomics and Outcomes Research in the areas of diagnosis, medical devices, and clinical, surgical and pharmacological intervention. The economic impact of health policy and health systems

organization also constitute important areas of coverage. The manuscript management system is completely online and includes a very quick and fair peer-review system, which is all easy to use. Visit http://www.dovepress.com/testimonials.php to read real quotes from published authors.

Submit your manuscript here: http://www.dovepress.com/clinicoeconomics-and-outcomes-research-journal 